\title{
An Empirical Analysis for 24 Asian Countries' Risks of Non-Gold Reserve Scale
}

\author{
Tao Ma ${ }^{1, a}$, Wenli Ding ${ }^{2, b}$, Ming $\mathrm{Li}^{3, \mathrm{c}}$ \\ ${ }^{1}$ The Institute of Applied Mathematics, Qujing Normal University, Qujing, China \\ ${ }^{2}$ School of Economics and Management, Yunnan Normal University, Kunming, China \\ ${ }^{3}$ Pan-Asia Business School, Yunnan Normal University, Kunming, China \\ a2977253061@qq.com, bingwl@163.com, ${ }^{\mathrm{c}} 1194789807 @$ qq.com \\ 亚洲 24 国非黄金储备规模风险实证分析 \\ 马涛 $1, \mathrm{a}$, 丁文丽 $2, \mathrm{~b}$, 李明 $3, \mathrm{c}$ \\ ${ }^{1}$ 曲靖师范学院应用数学研究所, 曲靖 655011 , 中国 \\ 2 云南师范大学经济与管理学院, 昆明 650500 , 中国 \\ 3 云南师范大学泛亚商学院, 昆明 650000 , 中国 \\ a2977253061@qq.com, bDingwl@163.com, ${ }^{c} 1194789807 @ q q . c o m$
}

\begin{abstract}
This paper tries to analyze the relationship among non-gold reserve, GDP and commodity trade export value. An empirical test is made by establishing a panel data model to test the above mentioned relationship of 24 Asian countries. The testing result shows that the non-gold reserve has a long-term co-integration relationship with each country's GDP and commodity trade export value. Meanwhile, foreign exchange reserve is the main body, the changing exchange rate will certainly bring risks to the value of reserve scale, the empirical analysis will help to pay attention to non-gold reserve scale's risk issues.
\end{abstract}

Keywords-Non-gold reserve, GDP, commodity export, Reserve scale risks, Panel data model

摘要一本文通过建立面板数据模型, 对亚洲 24 国 1990 年 至 2015 年间共 25 年的非黄金储备与 GDP 和货物贸易出口值 之间的关系进行实证分析，其中非黄金储备作为因变量, GDP 与货物贸易出口值为自变量。实证结果显示, 亚洲 24 国的非 黄金储备与各国 GDP 和货物贸易出口值之间存在长期的协整 关系。非黄金储备以外汇储备为主体, 汇率波动必然会对储 备规模的价值带来风险, 实证分析的结果有利于从另外的视 角关注非黄金储备规模的风险问题。

关键词一非黄金储备，GDP，商品出口，储备规模风险， 面板数据模型

\section{I. 引言}

国际货币基金组织（IMF）对各国储备分为两大 类：黄金储备和非黄金储备。其中，非黄金储备又称之 为总储备, 具体包括三个部分：第一，外汇储备; 第 二, 各国持有的 SDRs; 第三, 各国在 IMF 的储备头 寸。

2018 年 12 月, 中国的非黄金储备规模为 30918.81 亿美元。在非黄金储备中，外汇储备是主体，以中国总 储备为例, 2017 年和 2018 年中国的外汇储备占非黄金 储备资产的份额稳定在 $99.4 \%$ 左右。新中国成立以来, 中国外汇储备作为总储备的主体, 经历了从非常稀缺到 非常充足的转变。在改革开放之初的 1978 年, 中国的外
汇储备仅为 1.67 亿美元, 这一数据与新中国 1950 年 1.57 亿美元的外汇储备规模并无太大差别, 到 1980 年, 中国的外汇储备甚至为-12.96 亿美元, 外汇短缺十分明 显。随着改革开放的不断深入, 中国外汇储备规模逐步 攀升, 1996 年, 首次突破千亿美元大关, 达 1050.29 亿 美元; 2006 年, 又进一步突破万亿美元大关, 达 10663.44 亿美元; 2013 年至今, 中国外汇储备规模一直 保持在 3 万亿美元以上的水平, 成为名副其实的外汇储 备大国。如此巨大规模的储备规模, 汇率的少许波动都 有可能给外汇储备价值带来较大风险。

II. 亚洲 24 国非黄金储备、名义 GDP 与货物贸易 FOB 出口值简况

2015 年亚洲 24 国非黄金储备 (以下简写为 FXR) 、名义 GDP (以下简写为 GDP) 及货物贸易 FOB 出口（以下简写为 FOB）概况见表 1 。

中国外汇储备规模的不断扩大, 意味着其非黄金储 备规模的不断扩大，那么非黄金储备规模与中国的 GDP 总量以及中国货物出口贸易是否有明显相关的关系? 横 向扩展开来, 包含中国在内的亚洲部分国家的非黄金储 备规模与 GDP 和货物出口贸易这两大变量是否有显著的 关系? 这些关系是否有一些共性的地方可供参考, 以防 范储备规模过大而带来的风险问题, 是值得去深入研究 的一个方向。

当今，亚洲各国在 GDP 总量上差别很大，既有排名 世界第 2 的中国和排名世界第 3 的日本, 也有世界排名 比较靠后的一些国家, 诸如马尔代夫和不丹等国家。

国际贸易也是如此, 从各国国际收支平衡表的视 角, 各国货物贸易既是经常项下最为重要的项目, 也是 整个国际收支平衡表中最为重要的项目, 对各国总储备 有显著的影响。那么, 具体到各国货物贸易 FOB 出口值 与各国的总储备之间是否存在一定的规律性？

更宏观一点来看, 各国的 GDP 是否与各国的总储备 之间也存在一定的规律性? 
文章从总储备规模的视角, 以亚洲 24 国的总储备、 GDP 和货物贸易 FOB 出口值等三类数据为基础, 尝试
就各国 GDP 和 FOB 出口值对各国总储备的面板数据做 出实证分析。

表 12015 年亚洲 24 国相关经济数据概况表（单位: 百万美元）

\begin{tabular}{cccc}
\hline 国家 & $\mathbf{2 0 1 5}$ 年非黄金储备 & 2015 名义 GDP 总量 & 货物贸易 FOB 出口值 \\
\hline 中国 & 3345194 & 11181556 & 2284478 \\
日本 & 1207019 & 4124211 & 624787 \\
沙特阿拉伯 & 615985 & 646002 & 202237 \\
韩国 & 363149 & 1377873 & 526757 \\
印度 & 334311 & 2073002 & 267778 \\
新加坡 & 247534 & 292734 & 346638 \\
泰国 & 151266 & 395297 & 211028 \\
印度尼西亚 & 103268 & 858953 & 150358 \\
马来西亚 & 93979 & 296284 & 199158 \\
土耳其 & 92921 & 717932 & 143839 \\
菲律宾 & 73964 & 292451 & 58637 \\
黎巴嫩 & 38756 & 50807 & 3982 \\
科威特 & 28270 & 114079 & 54117 \\
孟加拉 & 27023 & 206531 & 31734 \\
巴基斯坦 & 17830 & 271050 & 22089 \\
阿曼 & 17543 & 64121 & 34734 \\
约旦 & 15162 & 37570 & 7849 \\
尼泊尔 & 7937 & 21314 & 720 \\
斯里兰卡 & 6543 & 81247 & 10470 \\
巴林 & 3387 & 31119 & 11200 \\
不丹 & 1103 & 2017 & 585 \\
老挝 & 1043 & 12561 & 2340 \\
马尔代夫 & 576 & 3120 & 144 \\
塞浦路斯 & 334 & 19330 & 1926 \\
\hline
\end{tabular}

III. 基于面板数据对亚洲 24 国 GDP 和货物贸易 FOB 出口值对总储备的影响的实证分析

\section{A. 模型建立}

为消除异方差等因素的影响，对各变量进行自然对 数处理, 由此建立的面板数据模型如下:

$$
L N F X R_{\mathrm{it}}=\alpha+\beta_{1} L N F O B_{\mathrm{it}}+\beta_{2} L N G D P_{\mathrm{it}}+\varepsilon_{\mathrm{it}}
$$

上述公式中, $\alpha$ 为常数项, $\beta_{1}$ 为 FOB 对 FXR 的影 响系数, $\beta_{2}$ 为 GDP 对 FXR 的影响系数, $\varepsilon$ 为随机误差 项。以下，本文将采用收集到的亚洲 24 国 1990 年至 2015 年的相关变量数据, 采用面板数据分析方法, 就 FOB 和 GDP 对 FXR 的影响情况进行分析。

\section{B. 平稳性检验}

由于面板数据具有时间序列的特征, 故在分析前要 先进行平稳性检验, 以避免产生伪回归现象, 常用的面 板数据平稳性检验为 LLC 检验和 ADF-Fisher 检验, 为 了能够全面地对变量平稳性情况进行检验, 本文同时采 用这两种检验方法。利用 EVIEWS 软件进行检验, 分别 对三个变量的平稳性情况进行检验, 所得到的结果汇总 如表 2 所示。

从表 2 的检验结果能够看出, 对于 LNFXR 的平稳 性检验, 其 LLC 检验的相应 $\mathrm{P}$ 值为 0.0001 , 小于 0.05 , 故拒绝 LNFXR 存在单位根的假设, 说明 LNFXR 是平稳 的, 而 ADF-Fisher 检验的相应 $\mathrm{P}$ 值为 0.1265 , 大于 0.05 , 故接受 LNFXR 存在单位根的假设, 说明 LNFXR 是非平稳的; 由于 ADF-Fisher 检验的结论表明其是非平
稳的, 因此可以认为 LNFXR 是非平稳的。对于一阶差 分 DLNFXR 的平稳性检验, 其 LLC 检验的相应 P 值为 0.0000 , 小于 0.05 , 说明 DLNFXR 是平稳的, 同时, 其 ADF-Fisher 检验的相应 $\mathrm{P}$ 值也为 0.0000 , 小于 0.05 , 说 明 DLNFXR 是平稳的, 两个检验方法的结论都显示是平 稳的, 因此可以认为 DLNFXR 是平稳的, 故 LNFXR 属 于一阶单整面板序列。按照同样的方法进行分析可知, 变量 LNFOB、LNGDP 都是非平稳的, 而一阶差分 DLNFOB、DLNGDP 都是平稳的, 这说明 LNFOB、 LNGDP 也都属于一阶单整面板序列。

表 2 平稳性检验结果

\begin{tabular}{ccccc}
\hline \multirow{2}{*}{ 变量 } & \multicolumn{2}{c}{ LLC 检验 } & \multicolumn{2}{c}{ ADF-Fisher 检验 } \\
\cline { 2 - 5 } & 统计量 & $\boldsymbol{P}$ 值 & 统计量 & $\boldsymbol{P}$ 值 \\
\hline LNFXR & -3.86779 & 0.0001 & 59.3313 & 0.1265 \\
DLNFXR & -19.3357 & 0.0000 & 330.970 & 0.0000 \\
LNFOB & -3.64320 & 0.0001 & 32.2148 & 0.9609 \\
DLNFOB & -18.3286 & 0.0000 & 312.162 & 0.0000 \\
LNGDP & 2.39680 & 0.9917 & 17.5558 & 1.0000 \\
DLNGDP & -13.0688 & 0.0000 & 241.615 & 0.0000 \\
\hline
\end{tabular}

经过以上检验可知, 变量 LNFXR、LNFOB、 LNGDP 都是一阶单整的, 属于同阶单整, 故接下来进 行协整检验分析。

\section{C. 协整检验}

运用 EVIEWS 软件, 利用 Kao Cointegration Test 检 验方法对 LNFXR 与 LNFOB、LNGDP 之间是否存在协 整关系进行检验。该检验方法的原假设是变量间不存在 协整关系, 若拒绝原假设, 则说明变量间存在协整关 系。

利用 EVIEWS 软件进行协整检验, 所得到的结果如 表 3 所示。 
表 3 协整检验结果

\begin{tabular}{ccc}
\hline \multicolumn{3}{c}{ Kao Residual Cointegration Test } \\
\hline ADF & t-Statistic & Prob. \\
& -6.168635 & 0.0000 \\
Residual variance & 0.085040 & \\
HAC variance & 0.085449 & \\
\hline 当假设为 “LNFXR 与 LNFOB、 LNGDP 之间不存在
\end{tabular}

协整关系” 时，Kao Cointegration Test 检验的统计量值 为-6.168635, 相应的 $\mathrm{P}$ 值为 0.0000 , 小于 0.05 , 故在 $5 \%$ 显著水平下拒绝该假设, 这说明 LNFXR 与 LNFOB、LNGDP 之间在长期存在协整关系, 即它们在 长期之间存在着一定的均衡发展关系。以下将进一步采 用回归分析方法就 FOB 和 GDP 对 FXR 的影响情况进行 估计分析。

\section{Hausman 检验}

对于面板数据的估计通常要选择合适的模型形式, 主要有固定效应模型和随机效应模型两种模型形式, 常 用的选择检验方法为 Hausman 检验, 该检验的原假设是 模型为随机效应模型，若在一定显著水平下拒绝原假 设, 则模型形式选择为固定效应模型, 若接受原假设就 为随机效应模型。

利用 EVIEWS 软件, 对本文所建立模型进行 Hausman 检验的结果如表 4 所示。

表 4 Hausman 检验结果

\begin{tabular}{cccc}
\hline \multicolumn{4}{c}{$\begin{array}{c}\text { Correlated Random Effects - Hausman Test } \\
\text { Test cross-section random effects }\end{array}$} \\
\hline Test Summary & Chi-Sq. Statistic & Chi-Sq. d.f. & Prob. \\
\hline Cross-section random & 45.096186 & 2 & 0.0000 \\
\hline
\end{tabular}

从表 4 结果可以看出, 该模型 Hausman 检验的统计 量值为 45.096186 , 其相应的 $\mathrm{P}$ 值为 0.0000 , 小于 0.05 , 故在 $5 \%$ 显著水平下应该拒绝模型为随机效应形式 的假设, 这说明本文所建立模型的估计应该选择固定效 应模型形式进行分析。

\section{E. 模型回归分析}

运用 EVIEWS 软件, 选择固定效应模型形式, 对所 建立的模型进行回归分析, 得到的结果如表 5 所示。

表 5 回归结果

\begin{tabular}{ccccc}
\hline Variable & Coefficient & Std. Error & t-Statistic & Prob. \\
\hline LNFOB & 0.789839 & 0.072182 & 10.94236 & 0.0000 \\
LNGDP & 0.555086 & 0.083350 & 6.659686 & 0.0000 \\
C & -4.477541 & 0.379776 & -11.78996 & 0.0000 \\
\hline R-squared & 0.958175 & Mean dependent var & 9.166704 \\
Adjusted R-squared & 0.956427 & S.D. dependent var & 2.425426 \\
S.E. of regression & 0.506289 & Akaike info criterion & 1.517356 \\
Sum squared resid & 153.2846 & Schwarz criterion & 1.702196 \\
Log likelihood & -447.4152 & Hannan-Quinn criter. & 1.589184 \\
F-statistic & 547.9889 & Durbin-Watson stat & 0.554482 \\
Prob(F-statistic) & 0.000000 & & \\
\hline
\end{tabular}

从模型的回归结果可以看出, 该模型估计的 $\mathrm{R}$ 方为 0.958175 , 拟合优度较高; $\mathrm{F}$ 统计量值为 547.9889 , 相应 的 $\mathrm{P}$ 值为 0 , 小于 0.05 , 这说明模型整体回归在 $5 \%$ 的水 平下是显著的。同时, 变量 $\mathrm{LNFOB}$ 的估计系数为 0.789839 , 该系数显著性检验的 $\mathrm{P}$ 值为 0.0000 , 小于 0.05 , 说明 LNFOB 对 LNFXR 存在显著的正向影响, 当 FOB 增长 $1 \%$ 时, 能够使 FXR 增长 $0.789839 \%$ 。变量 LNGDP 的估计系数为 0.555086 , 该系数显著性检验的 $\mathrm{P}$
值为 0.0000 , 小于 0.05 , 说明 LNGDP 也对 LNFXR 存在 显著的正向影响, 当 GDP 增长 $1 \%$ 时, 能够使 FXR 增长 $0.555086 \%$ 。

\section{IV. 结论与讨论}

本文通过建立 FOB 和 GDP 对 FXR 影响的面板数据 模型, 选择亚洲 24 国 1990 年至 2015 年的相关变量样本 数据进行分析可知, LNFXR 与 LNFOB、LNGDP 之间 在长期存在协整关系, 且 LNFOB 和 LNGDP 对 LNFXR 都存在显著的正向影响, 当 FOB 增长 $1 \%$ 时, 能够使 FXR 增长 $0.789839 \%$, 当 GDP 增长 $1 \%$ 时, 能够使 FXR 增长 $0.555086 \%$ 。

实证分析显示了 FOB 和 GDP 作为因变量都对 FXR 有显著的正向影响，但实证结果同时显示，FOB 显然对 FXR 的影响更大一些, 这也验证了实际国际金融领域出 口贸易对非黄金储备影响的巨大作用。

由于汇率波动等原因会对储备规模的实际价值带来比 较重大的影响, 国际储备规模本身并非越大越好, 非黄 金储备亦是如此, 合适的非黄金储备规模对于一国国民 经济发展具有至关重要的意义, 此实证分析结果为我国 和亚洲其他 23 国的非黄金储备规模提供了一个新的思考 视角, 因 GDP 增长和出口贸易增长而带来的外汇储备规 模增长问题具有长期协整关系, 可以通过增加进口支 出, 来缓解非黄金储备规模过大的问题, 以适度控制非 黄金储备规模，更为有效地控制国际储备的风险问题。

\section{致谢}

本项目受国家自然科学基金课题 “基于多国 CGE 模 型的人民币与东盟国家货币外汇市场福利效应研究” （编号：71363064）资助。

\section{通讯作者}

丁文丽, 云南师范大学经济与管理学院, 昆明 650500, 中国, dingwl@163.com

\section{参考文献}

[1] 厉以宁, “于我国外汇储备安全问题的思考” 中国流通经济, 2010, 24(4):4-7.

[2] 张荵, 凌盼盼. “我国黄金储备适度规模的实证研究” 西南金融, 2014(3):3-7.

[3] Gonqalo Pina, "International reserves and global interest rates" Journal of International Money and Finance 2017, 74: 371-385.

[4] 王益君. “外汇储备变动对通胀预期的影响及“阈值”效应” 中南财 经政法大学学报.2016(3):71-77.

[5] Pietro Cova, Patrizio Pagano, Massimiliano Pisani. "Foreign exchange reserve diversification and the 'exorbitant privilege': Global macroeconomic effects," Journal of International Money and Finance, 2016,67:82-101.

[6] 王增否, 张恒义. “中国外汇储备规模的生成机制及其影响因素研 究”华东经济管理, 2017(7).94-103.

[7] Abdullah Alam. "Crisis Transmission: Global Financial Crisis" Journal of Risk Analysis and Crisis Response, vol 2, issue 3, November 2012, 157 - 165.

[8] Dusan Marcek, Lukas Falat. "Volatility Forecasting in Financial Risk Management with Statistical Models and ARCH-RBF Neural Networks" Journal of Risk Analysis and Crisis Response, vol 4, issue 2, July 2014, $77-95$. 